\title{
Distribution of the reflection eigenvalues of a weakly absorbing chaotic cavity
}

\author{
C.W.J. Beenakker ${ }^{\mathrm{a}}$, P.W. Brouwer ${ }^{\mathrm{b}, *}$ \\ 'Instituut-Lorentz Untversitelt Leiden PO Box 95062300 RA Letden The Netherlands \\ ${ }^{\mathrm{b}}$ Laboratory of Atomic and Sold State Physics Cornell University Ithaca NY 14853-2501 USA
}

\begin{abstract}
The scattering-matrix product $S S^{\dagger}$ of a weakly absorbing medium is related by a unitary transformation to the time-delay matrix without absorption It follows from this relationship that the eigenvalues of $S S^{\dagger}$ for a weakly absorbing chaotic cavity are distributed according to a generalızed Laguerre ensemble (c) 2001 Elsevier Science B V All rights reserved
\end{abstract}

PACS $4225 \mathrm{Bs}, 0545 \mathrm{Mt}, 4225 \mathrm{Dd}, 7323-\mathrm{b}$

Keywords Chaotic scattering, Random-matrix theory, Optical absorption, Time-delay matrix

\section{Problem}

The purpose of this note is to answer a question raised by Kogan et al [1], concerning the statistics of the exgenvalues of the scattering-matrix product $S S^{\dagger}$ for an absorbing optical cavity with chaotic dynam1cs Without absorption the scattering matrix $S$ is an $N \times N$ unitary matrix, hence $S S^{\dagger} 1$ s simply the unit matrix $W_{l}$ th absorption the eigenvalues $R_{1}, R_{2}, \quad, R_{N}$ of $S S^{\dagger}$ are real numbers between 0 and 1 What is the probability distribution $P\left(\left\{R_{n}\right\}\right)$ of these reflection eigenvalues in an ensemble of chaotic cavities?

In principle, this problem can be solved by starting from the known distribution of $S$ in the absence of absorption (which is Dyson's curcular ensemble [2]), and incorporating the effects of absorption by

* Corresponding author Fax +1-607-255-6428

E-mall address brouwer@ccmr cornell edu (P W Brouwer) a fictitious lead [3] What has been calculated in this way is the distribution $P\left(\left\{R_{n}\right\}\right)$ for small $N$ [3] and the density $\rho(R)=\left\langle\sum_{n} \delta\left(R-R_{n}\right)\right\rangle$ for large $N$ $[4,5]$ These results have a complicated form, quite unlike those familiar from the classical ensembles of random-matrix theory [6] For example, in the presence of time-reversal symmetry the distribution for $N=2$ is given by Ref [3]

$$
\begin{aligned}
& P\left(R_{1}, R_{2}\right)=\left(1-R_{1}\right)^{-4}\left(1-R_{2}\right)^{-4} \\
& \quad \times \exp \left[-\gamma\left(1-R_{1}\right)^{-1}-\gamma\left(1-R_{2}\right)^{-1}\right] \\
& \quad \times\left|R_{1}-R_{2}\right|\left[\gamma^{2}\left(1-\mathrm{e}^{2 \gamma}+\gamma+\gamma \mathrm{e}^{2 \gamma}\right)\right. \\
& \quad+\gamma\left(R_{1}+R_{2}-2\right)\left(\frac{3}{2}-\frac{3}{2} \mathrm{e}^{2 \gamma}+2 \gamma+\gamma \mathrm{e}^{2 \gamma}+\gamma^{2}\right) \\
& \quad+\left(1-R_{1}\right)\left(1-R_{2}\right)\left(3-3 \mathrm{e}^{2 \gamma}+\frac{9}{2} \gamma\right. \\
& \left.\left.\quad+\frac{3}{2} \gamma \mathrm{e}^{2 \gamma}+3 \gamma^{2}+\gamma^{3}\right)\right],
\end{aligned}
$$


where $\gamma$ is the ratio of the mean dwell time $\tau_{\mathrm{d}}$ inside the cavity ${ }^{1}$ and the absorption time $\tau_{\text {n }}$

The situation is simpler for reflection from an absorbing disordered waveguide In the limit that the length of the waveguide goes to infinity, the distribution of the reflection eigenvalues becomes that of the Laguerre ensemble, aftc1 a transformation of var1ables from $R_{n}$ to $\lambda_{n}=R_{n}\left(1-R_{n}\right)^{-1} \geqslant 0$ The (unno1malized) distribution is given by $[7,8]$

$$
\begin{aligned}
P\left(\left\{\lambda_{l l}\right\}\right) \propto & \prod_{l<l}\left|\lambda_{i}-\lambda_{j}\right|^{\beta} \\
& \times \prod_{k} \exp \left[-\gamma(\beta N+2-\beta) \lambda_{k}\right],
\end{aligned}
$$

where now $\gamma=\tau_{\mathrm{s}} / \tau_{\mathrm{i}}$ contains the scattering time $\tau_{\mathrm{s}}$ of the disorder The integer $\beta=1(2)$ in the presence (absence) of time-reversal symmetry The eigenvalue density is given by a sum over Laguerre polynomials, hence the name "Laguerre ensemble" [6]

Kogan et al [1] used a maxımum entropy assumption [9] to argue that a chaotic cavity is also described by the Laguerre ensemble, but in the variables $R_{n}$ instead of the $\lambda_{n}$ 's Their maximum entropy disti1bution is

$$
P\left(\left\{R_{n}\right\}\right) \propto \prod_{l<1}\left|R_{l}-R_{l}\right|^{\beta} \prod_{l} \exp \left(-a R_{l}\right)
$$

The coefficient $a$ in the exponent is left undetermined ${ }^{2}$ Comparison with computer simulations gave good agreement for strong absorption, but not for weak absorption [1] This is unfortunate since the weak-absorption regime $(\gamma \ll 1)$ is likely to be the most interesting for optical experıments Although we know from the exact small- $N$ results [3] that no simple disti1bution exists in the entire range of $\gamma$, one might hope for a simple eigenvalue distribution for small $\gamma$ What is it?

\section{Solution}

Absorption with rate $1 / \tau_{1}$ is equivalent to a shift in frequency $\omega$ by an imaginary amount $\delta \omega=1 / 2 \tau_{\mathrm{a}}$

\footnotetext{
${ }^{1}$ The mean dwell time is related to the mean ficquency interval $\Delta$ of the cavity modes by $\tau_{d}-2 \pi / N \Delta$ so that $\gamma=2 \pi\left(\tau_{1} N \Delta\right){ }^{1}$ The definition of $\gamma$ used in Ref [3] differs by a factor $N$

${ }^{2}$ We have velified that the theory of Ref [3] agiees with Eq (3) fol stiong absorption $(\gamma \gg 1)$ with coefficient $a={ }_{2}^{1} \gamma \beta N$
}

If we denote by $S(0)$ the scattering matrix with absorption and by $S_{0}(\omega)$ the scattering matix without absorption, then $S(\omega)=S_{0}\left(\omega+1 / 2 \tau_{\uparrow}\right)$ For weak ab sorption we can expand

$$
\begin{aligned}
S_{0}\left(\omega+1 / 2 \tau_{\imath}\right) & \approx S_{0}(\omega)+\frac{1}{2 \tau_{\urcorner}} \frac{\mathrm{d}}{\mathrm{d} \omega} S_{0}(\omega) \\
& =S_{0}(\omega)\left[1-\frac{1}{2 \tau_{\urcorner}} Q(\omega)\right],
\end{aligned}
$$

where $Q=-1 S_{0}^{\dagger} \mathrm{d} S_{0} / \mathrm{d} \omega$ is the time-delay matix [10] Since $S_{0}$ is unitary, $Q$ is Hermitian The eigenvalues of $Q$, the delay times $\tau_{1}, \tau_{2}, \quad, \tau_{N}$, are real positive numbers $\mathrm{Eq} \mathrm{(4)} \mathrm{implies} \mathrm{that,} \mathrm{for} \mathrm{weak} \mathrm{absorption,}$

$S(\omega) S^{\dagger}(\omega)=S_{0}(\omega)\left[1-\frac{1}{\tau_{1}} Q(\omega)\right] S_{0}^{\dagger}(\omega)$

We conclude that the matrix product $S S^{\dagger}$ is related to the time-delay matrix $Q$ by a unitary transformation This relationship is a geneialization to $N>1$ of the result of Ramakrishna and Kumar [11] for $N=1$ (when the unitary tiansformation becomes a simple identity) Because a unitary transformation leaves the elgenvalues unchanged, one has $R_{n}=1-\tau_{l \prime} / \tau_{1}$, or equivalently, $\lambda_{n}=\tau_{1} / \tau_{n}$ (since $\lambda_{n} \rightarrow\left(1-R_{n}\right)^{-1}$ for weak absorption)

The probability distribution of the delay times in a chaotic cavity has recently been calculated, first for $N=1[12,13]$ and later for any $N[14,15]$ The corresponding distribution of the ieflection eigenvalues for weak absorption is a generalized Laguerre ensemble in the variables $\lambda_{n}$,

$$
\begin{aligned}
P\left(\left\{\lambda_{n}\right\}\right) \propto & \prod_{l<1}\left|\lambda_{l}-\lambda_{l}\right|^{\beta} \\
& \times \prod_{l} \lambda_{k}^{\beta N / 2} \exp \left[-\frac{1}{2} \gamma \beta N \lambda_{l}\right]
\end{aligned}
$$

The eigenvalue density is given in terms of generalszed Laguerre polynomials, hence the name The corresponding distribution of the reflection eigenvalues is

$$
\begin{aligned}
P\left(\left\{R_{n}\right\}\right) \propto & \prod_{l<1}\left|R_{l}-R_{l}\right|^{\beta} \\
& \times \prod_{l} \frac{\exp \left[-\frac{1}{2} \gamma \beta N\left(1-R_{l}\right)^{-1}\right]}{\left(1-R_{l}\right)^{2-\beta+3 \beta N / 2}}
\end{aligned}
$$

The first moment of this distribution is $N^{-1}\left\langle\sum_{n} R_{n}\right\rangle=$ $1-\gamma$, independent of $\beta$ One can check that $\mathrm{Eq}$ (7) 


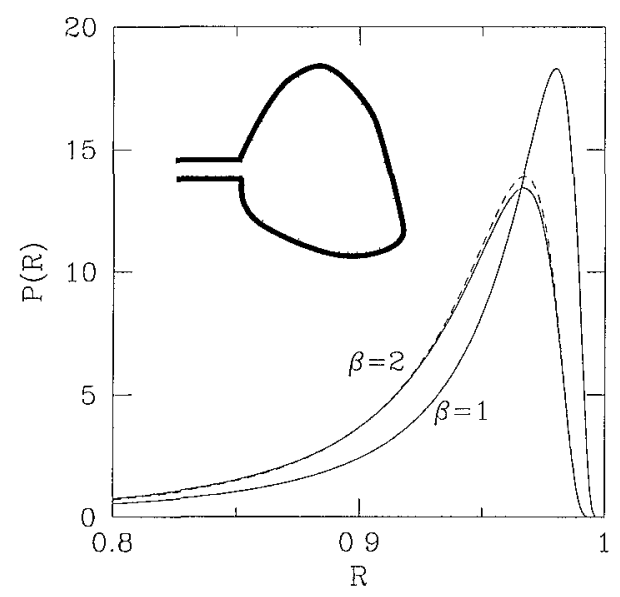

Fig. 1. Probability distribution of the reflectance of a weakly absorbing chaotic cavity that is coupled to the outside via a single-mode waveguide (inset). The solid curves have been computed from Eq. (8) for $\gamma=0.1$ (hence $\langle R\rangle=1-\gamma=0.9$ ). The dashed curve is the exact $\beta=2$ result from Eq. (9).

is the small- $\gamma$ asymptote of the exact result (1) for $N=2, \beta=1$.

In the case $N=1$ of a single scattering channel, the distribution (7) reduces to

$$
\begin{aligned}
P(R)= & \frac{(\gamma \beta / 2)^{1+\beta / 2}}{\Gamma(1+\beta / 2)}(1-R)^{-2-\beta / 2} \\
& \times \exp \left[-\frac{1}{2} \gamma \beta(1-R)^{-1}\right],
\end{aligned}
$$

including the normalization constant. We have plotted this function in Fig. 1 for $\gamma=0.1$ and $\beta=1,2$. It is totally different from the exponential distribution $P(R) \propto \exp (-a R)$ of Ref. [1]. For comparison, we have also included in Fig. 1 the exact $N=1$ result [16] (which is known only for $\beta=2$ ):

$$
\begin{aligned}
P(R)= & (1-R)^{-3} \exp \left[-\gamma(1-R)^{-1}\right] \\
& \times\left[\gamma\left(\mathrm{e}^{\gamma}-1\right)+\left(1+\gamma-\mathrm{e}^{\gamma}\right)(1-R)\right] .
\end{aligned}
$$

It is indeed close to the small- $\gamma$ asymptote (8).

\section{Conclusion}

Summarizing, the distribution of the reflection eigenvalues of a weakly absorbing chaotic cavity is the generalized Laguerre ensemble (6) in the parameterization $\lambda_{n}=R_{n}\left(1-R_{n}\right)^{-1}$. The Laguerre ensemble
(3) in the variables $R_{n}$, following from the maximum entropy assumption [1], is only valid for strong absorption. For intermediate absorption strengths the distribution is not of the form of the Laguerre ensemble in any parameterization, cf. Eq. (1). In contrast, the distribution of a long disordered waveguide is the Laguerre ensemble (2) for all absorption strengths.

The relationship between the reflection eigenvalues for weak absorption and the delay times implies that the delay times $\tau_{n}$ for reflection from a disordered waveguide of infinite length are distributed according to Eq. (2) if one substitutes $\gamma \lambda_{n} \rightarrow \tau_{s} / \tau_{n}$. The implications of this Laguerre ensemble for the delay times will be discussed elsewhere.

\section{Acknowledgements}

Correspondence with E. Kogan and P.A. Mello has stimulated us to look into this problem. This research was supported by the "Nederland-se organisatie voor Wetenschappelijk Onderzoek" (NWO), by the "Stichting voor Fundamenteel Onderzoek der Materie" (FOM), and by the National Science Foundation (Grant numbers DMR 94-16910, DMR 96-30064, DMR 97-14725).

\section{References}

[1] E. Kogan, P.A. Mello, H. Liqun, Phys. Rev. E 61 (2000) 17.

[2] C.W.J. Beenakker, Rev. Mod. Phys. 69 (1997) 731.

[3] P.W. Brouwer, C.W.J. Beenakker, Phys. Rev. B 55 (1997) 4695.

[4] C.W.J. Beenakker, Phys. Rev. Lett. 81 (1998) 1829.

[5] C.W.J. Beenakker, in: J.-P. Fouque (Ed.), Diffuse Waves in Complex Media, NATO Science Series C531, Kluwer, Academic Publishers, Dordrecht, 1999.

[6] M.L. Mehta, Random Matrices, Academic Press, New York, 1991.

[7] C.W.J. Beenakker, J.C.J. Paasschens, P.W. Brouwer, Phys. Rev. Lett. 76 (1996) 1368.

[8] N.A. Bruce, J.T. Chalker, J. Phys. A 29 (1996) 3761.

[9] P.A. Mello, H.U. Baranger, Waves Random Media 9 (1999) 105.

[10] Y.V. Fyodorov, H.-J. Sommers, J. Math. Phys. 38 (1997) 1918.

[11] S.A. Ramakrishna, N. Kumar, Phys. Rev. B 61 (2000) 3163.

[12] Y.V. Fyodorov, H.-J. Sommers, Phys. Rev. Lett. 76 (1996) 4709. 
[13] V A Gopar, P A Mello, M Buttıke,, Phys Rev Lett 77 (1996) 3005

[14] P W Biouwer, KM Frahm, CW J Beenakker, Phys Rev Lett 78 (1997) 4737
[15] P W Brouwer, KM Frahm, C W J Beenakker, Waves Random Media 9 (1999) 91

[16] P W Brouwer, unpublished calculation, 1996 